\title{
Kimberley Christine Patton, John Stratton Hawley, éds., Holy Tears. Weeping in the Religious Imagination
}

Princeton \& Oxford, Princeton University Press,

2004, XII+317 p.

André Padoux

\section{(2) OpenEdition}

Journals

Édition électronique

URL : http://journals.openedition.org/assr/4015

DOI : 10.4000/assr.4015

ISSN : $1777-5825$

Éditeur

Éditions de l'EHESS

Édition imprimée

Date de publication : 1 décembre 2006

Pagination : 115-283

ISBN : 2-7132-2124-2

ISSN : 0335-5985

Référence électronique

André Padoux, « Kimberley Christine Patton, John Stratton Hawley, éds., Holy Tears. Weeping in the Religious Imagination », Archives de sciences sociales des religions [En ligne], 136 | octobre - décembre 2006, document 136-84, mis en ligne le 14 février 2007, consulté le 21 septembre 2020. URL : http:// journals.openedition.org/assr/4015; DOI : https://doi.org/10.4000/assr.4015

Ce document a été généré automatiquement le 21 septembre 2020.

(C) Archives de sciences sociales des religions 


\section{Kimberley Christine Patton, John Stratton Hawley, éds., Holy Tears. Weeping in the Religious Imagination}

Princeton \& Oxford, Princeton University Press, 2004, XII+317 p.

André Padoux

1 "Qui fera l'histoire des larmes?", se demandait Roland Barthes (Fragments d'un discours amoureux, 1977). Les larmes ont, en effet, été bien moins étudiées que d'autres sécrétions corporelles, le sang et le sperme notamment. Or, elles sont importantes car elles touchent au rapport entre le corps et l'intériorité. Il y a un mystère de la production des larmes : "Jaillies du plus profond de l'être », elles apparaissent comme médiatrices entre l'homme et la société, entre l'être humain et le divin. Relevant du psychologique, du social et du culturel, elles sont d'un grand intérêt : les articles dont on va rendre compte en témoignent. Ce volume se situe dans un ensemble d'études poursuivies ces dernières années aux États-Unis, marquées notamment par l'ouvrage de Tom Lutz, Crying. The Cultural and Natural History of Tears (New York, 1999), considéré là-bas comme une sorte de classique sur le sujet. Issu des réunions annuelles, en 2001 et 2002, de l'American Academy of Religion, dont le thème était le rôle des larmes dans le domaine religieux, il en reprend plusieurs exposés en en ajoutant d'autres, dont certains s'expliquent par les circonstances du moment, car la première de ces deux réunions, s'était tenue deux mois à peine après le 11 septembre 2001 dans une atmosphère de particulière émotion, que l'on retrouve dans l'introduction comme dans la dernière contribution du volume.

2 Patton et Hawley précisent dans leur introduction que c'est de divers aspects du rôle joué, dans le contexte social et historique, par les larmes, pleurs et lamentations « in the life of religion » que traitera cet ouvrage : des larmes de l'ascèse, de la dévotion, de l'invocation, de la componction, ou de la joie mystique - des larmes de Dieu, même. Les larmes étant bien souvent versées dans des circonstances particulières, fixées d'avance, et notamment rituelles, la question se pose de leur caractère spontané ou 
conventionnel, privé ou public. Les pleurs rituels peuvent avoir un rôle social, un aspect moral, politique, cosmologique même, ce qui pose le problème du rapport des larmes versées à l'émotion éprouvée. L'aspect sexué des larmes est aussi évoqué : elles sont souvent l'affaire des femmes plutôt que des hommes. Patton et Hawley notent également qu'en anglais on peut distinguer entre "weeping", verser des larmes, souvent silencieuses et "crying", qui suppose des pleurs bruyants, cris ou lamentations, parfois prescrits, parfois aussi condamnés au profit d'une effusion paisible.

3 Le premier article, de G.L. Ebersole, sur les « Poetics and politics of ritualized weeping » dans l'ancien Japon, ne concerne pas les pleurs religieux, mais certaines circonstances sociales où des larmes étaient prescrites. Tel n'était pas le cas dans le monde aztèque envisagé par Kay Almere Read, où les larmes sont versées lors de rites agricoles ou guerriers, étant liées à une vision cosmique, cela dans un pays sec où l'eau est symbole ou métaphore de tout ce qui est bon. Il y a là un langage puissant et efficace des larmes: "weeping served as morally productive acts that created cosmic and social goods».

4 Il est ensuite question des larmes rituelles dans la Grèce ancienne et, accessoirement, moderne. Le bref article de G.O.L. Lynch Pollock est illustré de reproductions de lécythes, vases utilisés dans les rites funéraires, ornés de scènes de deuil et de pleurs rituels. Ces pleurs restent de rigueur de nos jours dans les villages grecs, signes de deuil inscrivant la douleur privée dans le contexte de la communauté et même plus généralement, pense l'auteur, la mort dans le flot de la vie.

5 En se référant aux articles précédant le sien, lequel concerne les pleurs des gopis, les vachères amoureuses du dieu Krishna, tels que les conte le poète mystique Surdas, J.S. Hawley pose dès l'abord que quatre traits caractérisent les pleurs religieux : ils ne sont pas spontanés, mais prescrits ou joués «enacted»; ils ne font pas obstacle à la communication, mais au contraire la favorisent; ils sont généralement féminins et interviennent fréquemment à propos d'une mort. Il est vrai que ces traits se retrouvent souvent, mais on ne les voit précisément guère dans le cas des gopis. Celles-ci sont en effet profondément affligées par l'absence du dieu qu'elles aiment : leurs pleurs sont spontanés. Sans doute, en pleurant expriment-elles leur peine et la font-elles partager, et enfin ce sont des femmes. Mais elles pleurent un absent, non pas un mort, le dieu Krishna étant éternel. Si toutefois la théorie n'est pas ici justifiée, ce que Hawley dit de la virahabhakti, la dévotion au dieu absent, n'est pas inintéressant. Bien d'autres exemples, parfois curieux, de pleurs rituels ou dévots en Inde auraient pu être mentionnés, mais ce recueil avait nécessairement ses limites. On pourrait faire la même observation à propos des pleurs versés par le pèlerin chinois Huian-Tsang quand il retrouve en Inde les traces laissées par le Bouddha dont traite (brièvement) M.D. Eckel, qui a choisi malheureusement un incident mineur et peu probant, alors qu'il y a une importante tradition et une doctrine des pleurs de compassion dans le bouddhisme, qui eussent pu être, non pas certes traitées, mais au moins abordées.

6 Avec l'étude de J.K. Olupona et S. Ajibadé sur les larmes versées lors du mariage chez les Oxu-Yoruba du Nigeria, ce sont de pleurs faisant partie du cérémoniel matrimonial, donc ritualisés, prescrits, qu'il s'agit. Mais ils expriment aussi la profonde émotion vécue par la jeune mariée lors de ce rite de passage, cependant que, par leur caractère collectif, ces pleurs (la mariée n'étant pas seule à pleurer), les danses et les poèmes récités donnent une expression à des réalités de la vie familiale et collective des Oxu- 
Yoruba. On a vu dans d'autres articles de ce volume ce double aspect de la «metaphor of tears and weeping " : il lui est essentiel et fait sa richesse.

7 Deux contributions concernent l'islam. Dans la première, "Weeping in classical Sufism », W.C. Chittick rappelle les cas, rares, où le Coran mentionne les larmes et renvoie au Kashf al-asrar, commentaire du Coran en persan, datant du XIII ${ }^{\mathrm{e}}$ siècle, dont il cite quelques passages. Le deuxième article, de A. Bard, «Tears and transformation in South Asian Majlis Poetry ", est bien plus intéressant. Il traite des pleurs versés pendant les majlis, les assemblées de fidèles shiites lors du Moharram où sont récitées des poésies évoquant la mort de l'imam Husain à Karbala. Ces pleurs sont rituels, prescrits, mais, nés de l'évocation à la fois personnelle et collective d'un fait dramatique intensément ressenti, ils sont aussi émotionnellement vécus. Les majlis sont célébrés en Inde depuis le $\mathrm{XVI}^{\mathrm{e}}$ siècle, mais c'est au XIX ${ }^{\mathrm{e}}$ siècle que, dans le nord du pays, ils ont pris de l'ampleur et que la poésie évoquant Husain y a tenu une place notable. A. Bard, citant de ces poèmes en urdu et persan, évoque ces pleurs joués et pourtant passionnés, où les larmes sont à la fois l'expression visible d'un état intérieur et ce qui provoque cet état : il y a une efficacité aussi bien intérieure qu'extérieure de la mise en scène de la douleur - une féconde ambiguïté des larmes.

les larmes dans la tradition juive (talmudique, essentiellement) qu'envisage H.W. Basser, "Weeping in Jewish Sources». On pense tout de suite à ce propos aux lamentations de Jérémie, aux pleurs de Rachel ou aux larmes versées après la destruction du Temple, occasion où d'ailleurs Dieu lui-même aurait pleuré, ce dernier thème étant très présent dans la littérature talmudique. La tradition accorde une grande importance aux larmes, les recommande dans certaines circonstances, mais elle en limite et en règle l'usage : les pleurs incontrôlés sont dangereux, il ne faut pas s'attendrir sur soi. Les larmes peuvent toutefois être comme un autel du sacrifice: Basser cite Rachi à cette occasion. Il termine en se référant à la Kabale, en citant un récit où les lamentations apparaissent comme ayant un pouvoir contraignant et quasi magique.

9 Les trois contributions suivantes concernent le domaine chrétien. Avec d'abord le cas, examiné par S. Bhattacharji, de la mystique anglaise Margery Kempe (1373 ?-1438), à qui l'évocation des souffrances du Christ arrachait non seulement des pleurs mais de bruyantes lamentations qui, quand elles se produisaient en public, perturbaient les célébrations religieuses auxquelles elle assistait. Si elle en était parfois honteuse, elle pensait que ces manifestations publiques avaient une valeur exemplaire, encourageant d'autres à la dévotion. Des évènements de la vie quotidienne pouvaient aussi provoquer ces pleurs. On est loin, là, des larmes silencieuses de l'effusion mystique. Mais on sait que d'autres mystiques, Angèle de Foligno par exemple, avaient parfois des comportements analogues. S.B. attire l'attention sur le fait que Margery Kempe ne pleurait pas seulement à cause des souffrances du Christ, mais aussi par participation à son humanité : si ses pleurs étaient versés pour ses péchés et sur les douleurs de la Crucifixion, ils l'étaient aussi à cause de la joie qu'elle éprouvait à sentir la présence humaine du Christ. C'est une attitude que l'on trouve d'une façon voisine chez Angèle de Foligno, qui avait d'ailleurs, comme elle, fait l'expérience de l'amour humain.

D. Apostolos-Cappadona traite de la représentation artistique des larmes, en prenant l'exemple de celles de sainte Marie-Madeleine, figure symbolique née de la fusion des personnes de Marie de Béthanie, sœur de Lazare, qui arrosa de ses larmes et d'un onguent précieux les pieds du Christ (qui avait pleuré cette mort) et de Marie de 
Magdala qui vint avec des aromates à son sépulcre et le trouva vide : deux anges lui dirent « Femme, pourquoi pleures-tu ? » (Jean 20.13). Se développa alors en Occident, la doctrine de la grâce des larmes, gratia lacrimarum. Les larmes n'étaient dès lors plus seulement celles du deuil et de la componction, mais une réponse humaine - qui peut être elle-même une grâce : le "don des larmes »- à la grâce divine. L'auteur étudie ainsi la représentation des larmes de la Madeleine telle qu'on la voit dans la Descente de Croix de Rogier van der Weyden, ou dans la Pieta d'Avignon, pour finir par la Femme en pleurs de Picasso, datant de 1937, image frappante des larmes de désespoir versées par une femme devant les douleurs du monde. (À noter que le thème du don des larmes au Moyen Âge a fait l'objet d'une intéressante étude par P. Nagy, Albin Michel, 2000.)

11 K. Ware envisage rapidement la question du don des larmes dans la spiritualité de l'Église d'Orient en se référant surtout à Jean Climaque, qui distinguait entre les larmes amères du chagrin et les larmes douces de la joie. Mais il y a aussi des larmes naturelles et d'autres qui sont surnaturelles, et sont une manifestation de la grâce en même temps que son effet. L'auteur mentionne dès le début la belle étude de Myrrha Lot-Borodine, "Le mystère du "don des larmes" dans l'orient chrétien ", rééditée dans le volume La douloureuse joie (Abbaye de Bellefontaine, 1974). Le même sujet, « Holy tears in Eastern Christianity ", est repris sur un ton plus personnel, vécu, par K. Patton.

Le volume se termine, d'une façon qui peut surprendre mais qu'expliquent les évènements de l'époque, par la transcription d'un entretien de Hawley et Patton avec Betsy Parker, pasteur de l'Église épiscopalienne, qui se trouvait être « Head Chaplain » au centre médical créé à Ground Zero après la destruction du World Trade Center, lieu où les larmes ne firent pas défaut. C'est la douleur et les larmes des familles des victimes de l'attentat comme de ceux qui, dans les décombres, ramassaient des restes humains rassemblés et bénis ensuite par des religieux de diverses croyances, qu'ont évoqués Betsy Parker et ses deux interlocuteurs. Du vécu des larmes, d'une douleur partagée, allégée peut-être par les pleurs, dont la nature, la source, l'effet sont ici encore examinés. Étrangement, B. Parker et plusieurs participants aux travaux de déblaiement disent avoir perçu, à un moment, un son resté inexpliqué, «a deep frightening moaning sound ", qu'ils ont interprété comme les pleurs de Dieu devant le malheur des hommes; "I knew that I was hearing God weeping». Intéressante survivance d'une ancienne notion, preuve aussi de la prégnance toujours vivante des larmes, médiatrices entre l'être humain et ce qu'il éprouve comme le transcendant. Par les références et les faits décrits comme par les réflexions qui les accompagnent, l'ouvrage mérite l'attention. Ses notes contiennent une assez abondante et utile bibliographie. 